\title{
Analyse théorique d'un rideau ancré
}

\section{S. BARUSSAUD}

Ingénieur Conseil, Société ESF, BP 56 , 94290 Villeneuve-le-Roi
Cet article analyse le comportement d'une paroi ancrée à l'aide d'un modèle aux éléments finis.

Les résultats obtenus à l'aide de ce modèle sont discutés et comparés avec les hypothèses de calcul des méthodes classiques.

On montre que l'hypothèse de Winkler, c'est-à-dire la modélisation du sol par un module de réaction, permet de prévoir les efforts dans la paroi, mais nécessite des aménagements pour permettre une prédiction des déplacements.

Enfin, il est montré que l'utilisation d'une transformation de Fourier de l'équation de Winkler permet d'obtenir une définition du module de réaction compatible avec la théorie de l'élasticité du milieu semi-infini.

\section{Theoritical analysis of a tied back wall}

This paper analyses the beharior of a tied back wall by mean of a finite element model.

The results obtained are discussed and compared to the asumptions on which classical methodes of desining are based. It is shown that the Winkler Hypothesis of subgrade reaction modulus may be used to predict stresses in the wall but need to be modified in order to predict wall displacements.

Finally it is shown that using Fourier's transform of the Winkler equation allows to define a subgrade reaction modulus according to the theory of an elastic half-space. 


\section{NOTATIONS}

\begin{tabular}{|c|c|c|}
\hline B & : & largeur de la paroi \\
\hline C & : & cohésion du sol \\
\hline$E_{p}$ & & module d'Young de la paroi \\
\hline $\mathrm{E}_{\mathrm{s}}$ & : & module d'Young du sol \\
\hline F & & force horizontale \\
\hline$g(z)$ & & déplacement du sol \\
\hline$G(f)$ & : & transformée de Fourier de g \\
\hline f & & inverse d'une longueur \\
\hline$f_{0}$ & & valeur de référence de $f$ \\
\hline $\mathrm{h}$ & & hauteur de la paroi \\
\hline$I_{p}$ & & inertie de la paroi \\
\hline K & & module de réaction \\
\hline$l_{0}$ and $l_{0}^{*}$ & & longueur de référence \\
\hline $\mathrm{M}(\mathrm{z})$ & : & moment fléchissant \\
\hline $\mathrm{p}(\mathrm{z})$ & & pression de contact horizontale \\
\hline$y(z)$ & & déplacement de la paroi \\
\hline$Y(f)$ & & transformée de Fourier de y \\
\hline z & & coordonnée de profondeur \\
\hline$\alpha$ & : & coefficient sans dimension \\
\hline$\varphi$ & & angle de frottement interne du sol \\
\hline$\lambda$ et $\mu$ & & coefficients sans dimension \\
\hline$\gamma_{s}$ & & poids spécifique du sol \\
\hline$\gamma_{p}$ & & poids spécifique de la paroi \\
\hline$\sigma_{\mathrm{h}}$ & : & contrainte horizontale \\
\hline$\sigma_{v}$ & : & contrainte verticale \\
\hline$v$ & & coefficient de Poisson \\
\hline$\Pi$ & : & transformée de Fourier de $\mathrm{p}(\mathrm{z})$ \\
\hline$\Omega$ & & transformée de Fourier de $\mathrm{M}(\mathrm{z})$ \\
\hline
\end{tabular}

\section{Introduction}

Les méthodes de calcul des rideaux ancrés peuvent être classées en trois catégories :

a) les méthodes aux états-limites de plastification fondées sur les distributions de contraintes du type Rankine ou Coulomb ;

b) l'approche de Winkler qui représente le sol par des ressorts indépendants (méthodes au module de réaction) :

c) la méthode des éléments finis qui permet de représenter le massif de sol dans son ensemble ainsi que le soutènement qui lui est associé.

Le but de cet article est d'examiner quelques-unes des hypothèses sur lesquelles reposent ces méthodes et, en particulier, l'hypothèse du module de réaction.

Les résultats sur lesquels s'appuient les développements ci-après sont tirés d'un modèle utilisant les éléments finis pour simuler une paroi ancrée par un lit de tirants précontraints.

Le comportement de la paroi et des tirants est purement élastique tandis que le sol est élasto-plastique avec un critère de rupture type Mohr-Coulomb.

Quatre phases de travaux ont été simulées :

- Phase 1 : Le sol est horizontal à la cote 21. Une paroi moulée est mise en place sans modification de l'état de contrainte.

- Phase 2 : A l'aval de la paroi, le terrain est excavé jusqu'à la cote 17 .

- Phase 3 : Mise en place et précontrainte des tirants.

- Phase 4 : L'excavation est poursuivie jusqu'à la cote 10,5 .

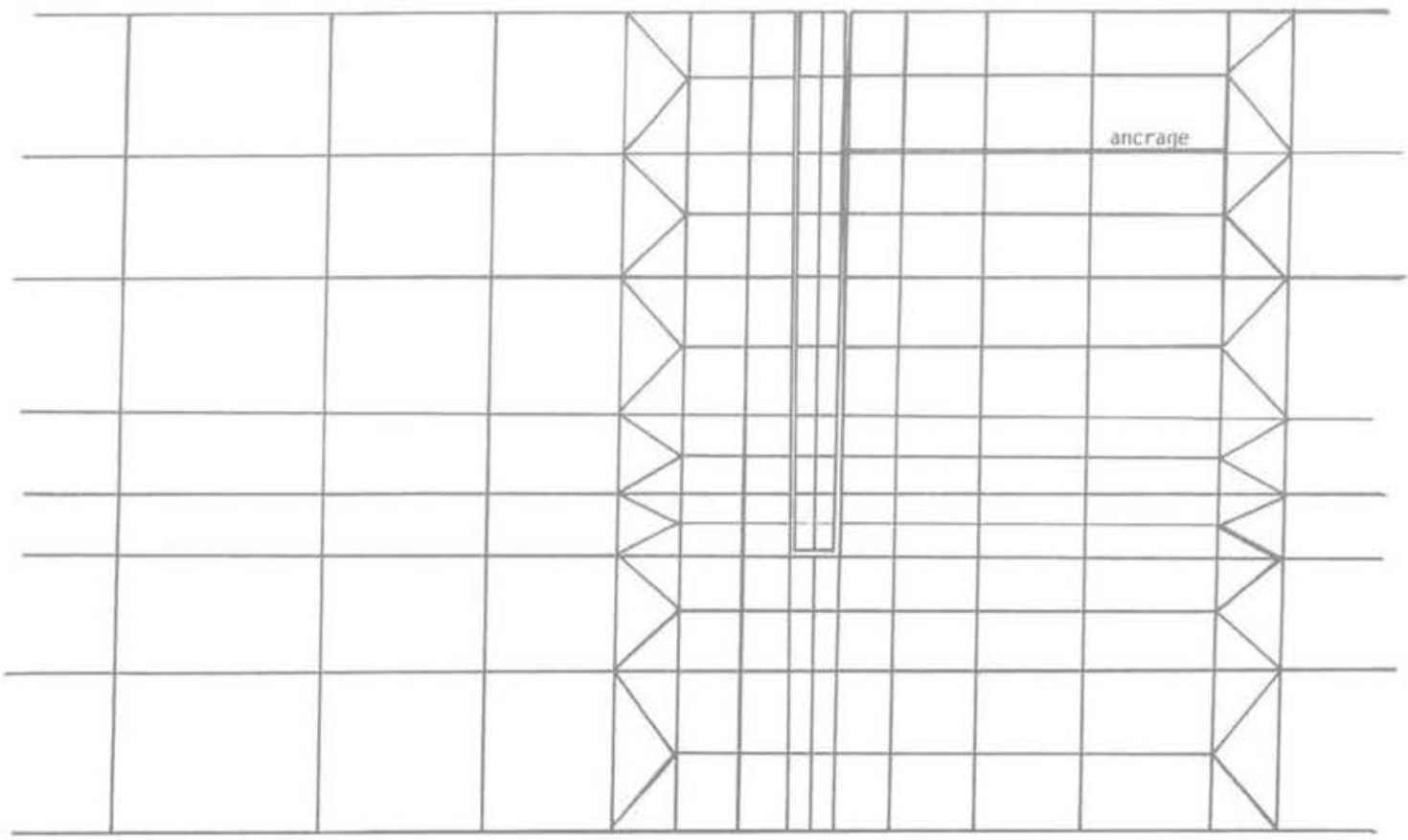



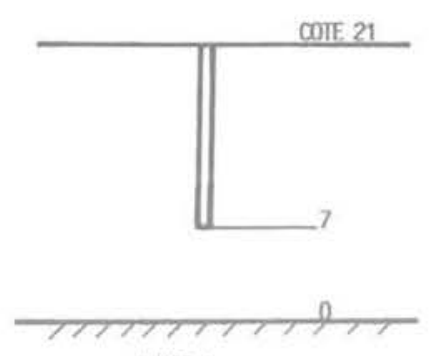

PAnc 1

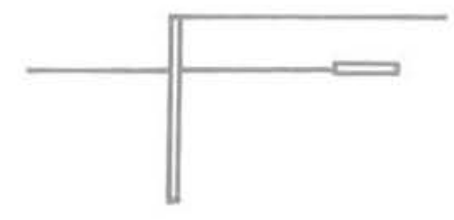

PIAE 3

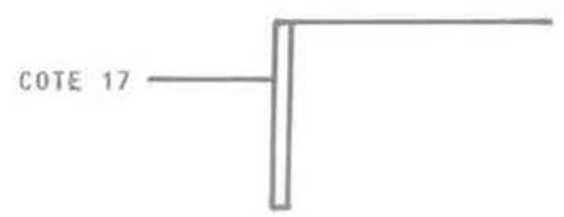

PASE 2

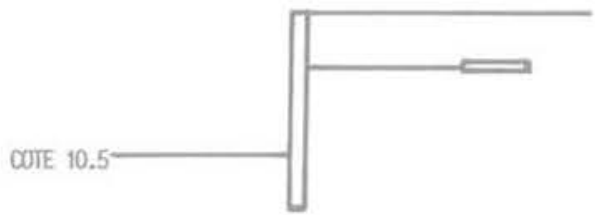

PALE 4

FIG.2 Phasage des terrassements.

Steps of construction sequence.

Les différents cas étudiés sont regroupés dans le tableau I.

Test de référence T 100:

Valeurs des paramètres :

- sol : $E_{\mathrm{s}}=10^{4} \mathrm{kPa} \quad v=0,33 \quad \gamma_{\mathrm{s}}=20 \mathrm{kN} / \mathrm{m}^{3}$

- paroi : $\mathrm{B}=1 \mathrm{~m} \quad \mathrm{~h}=14 \mathrm{~m}$

- Ep $=2.10^{7} \mathrm{kPa} \quad v=0,33 \quad \gamma_{\mathrm{p}}=23 \mathrm{kN} / \mathrm{m}^{3}$

Tirant en acier : Module d'Young $\mathrm{E}=2,2.10^{8} \mathrm{kPa}$

Surface relative d'acier : $2.10^{-3} \mathrm{~m}^{2} / \mathrm{m}$

Précontrainte : $\mathrm{F}=200 \mathrm{kN} / \mathrm{m}$

Contact sol/paroi rugueux

\section{2 \\ Résultats du modèle aux éléments finis}

Parmi les résultats obtenus lors de chacune des phases, on s'intéressera plus particulièrement aux paramètres suivants :

- déplacement du mur :

- pression de contact contre la paroi ;

- moments fléchissants dans la paroi ;

- extension des zones plastifiées dans le sol.

\section{TABIEAUI Cas étudiés.}

\begin{tabular}{|c|c|c|c|c|}
\hline Test $n^{\circ}$ & Largeur du modèle (m) & Type de contact" & F & Autres paramètres \\
\hline 000 & 60 & Rugueux & non ancré & $\mathrm{Ep}=\mathrm{Es}$ (pas de paroi) \\
\hline 001 & 60 & Rugueux & non ancré & \\
\hline 100 & 60 & Rugueux & 200 & Test de référence \\
\hline 101 & 60 & Rugueux & 400 & - \\
\hline 103 & 60 & Rugueux & 800 & - \\
\hline 110 & 100 & Rugueux & 200 & - \\
\hline 200 & 100 & Rugueux & non ancré & Ep = Es (pas de paroi) \\
\hline 201 & 100 & Lisse & non ancré & $\mathrm{Ep}=10^{5}$ \\
\hline 202 & 100 & Lisse & 200 & \\
\hline 210 & 100 & Lisse & 200 & \\
\hline 211 & 100 & Lisse & 200 & Zone d'ancrage modifiée \\
\hline 212 & 100 & Lisse & 200 & . \\
\hline 310 & 100 & Rugueux & 200 & $\begin{array}{c}\text { Critère de plasticité } \\
\text { de Coulomb: } C=0 \varnothing=35^{\circ}\end{array}$ \\
\hline 311 & 100 & Lisse & 200 & \\
\hline
\end{tabular}




\section{Déplacements du mur}

L'influence des limites latérales du modèle a été étudiée à l'aide des modèles T100 et T110 dont les dimensions horizontales sont respectivement de 60 et de $100 \mathrm{~m}$ (Tab. I).

Les écarts obtenus pour les déplacements sont regroupés dans le tableau II.

L'accroissement des dimensions du modèle a une influence notable sur les déplacements de la paroi lors des phases d'excavation.

En revanche, les déplacements associés à la mise en tension des tirants semblent indépendants de la taille du modèle.

TABLEAUII Déplacement horizontal de la paroi (en mm) Influence de la taille du modèle.

\begin{tabular}{l|c|c|c}
\cline { 2 - 4 } & T100 & T110 & Différence \\
\cline { 2 - 4 } Phase 2 & & & \\
Tête & 10,7 & 14,3 & 3,6 \\
Pied & 26,1 & 28,9 & 2,8 \\
\hline Phase 3 & & & \\
Tête & 4,2 & 7,9 & 3,7 \\
Pied & 26,6 & 29,4 & 2,8 \\
\hline Phase 4 & & & \\
Tête & 29,2 & 36,7 & 7,5 \\
Pied & 60,3 & 62,8 & 2,5 \\
\hline
\end{tabular}

Le type de contact entre le sol et la paroi (contact rugueux ou contact lisse) influence grandement les déplacements horizontaux ainsi que la prise en compte de la plastification du sol (Fig. 3).

\section{2}

\section{Pressions de contact sol/paroi}

Les pressions de contact semblent pratiquement indépendantes des dimensions du modèle. En revanche, le type de contact (lisse ou rugueux) est très important, en particulier, juste en dessous du fond de fouille (Fig. 4).

\section{3}

\section{Moments fléchissants}

Les dimensions du modèle sont sans influence sur les moments fléchissants de la paroi.

Pour un sol purement élastique (T110 et T210), on constate que les moments fléchissants sont nettement plus élevés pour un contact lisse sol/paroi que pour un contact rugueux.

Cependant, la plastification du sol (T310 et T311) tend à réduire ces différences (Fig. 5).

\section{4}

\section{Plastification du sol}

Avec le même critère de Mohr-Coulomb $(\mathrm{C}=\mathrm{O}$ et $\varphi=35^{\circ}$ ), on observe que les zones plastifiées sont nettement plus étendues dans le cas d'un contact lisse que dans celui d'un contact rugueux.

Les zones plastifiées s'étendent principalement devant la paroi, sous le fond de fouille et à l'arrière de la zone d'ancrage. L'état de poussée Ka n'est pas atteint à l'arrière du mur (Fig. 6).

\section{3}

\section{Relation entre moments fléchissants et rigidité}

La rigidité de la paroi est définie par le terme $E_{p} I_{p} / E_{s}$ dans lequel $E_{p}$ est le module d'Young de la paroi ef $I_{p}^{p}$ son inertie. $E_{s}$ est le module d'Young du sol.

A partir d'un modèle simulant une paroi soumise à des efforts horizontaux en tête, on obtient la relation suivante :

$$
\mathrm{M}_{\max }=\lambda \mathrm{F}\left(\mathrm{E}_{\mathrm{p}} \mathrm{I}_{\mathrm{p}} / \mathrm{E}_{\mathrm{s}}\right)^{1 / 3}
$$

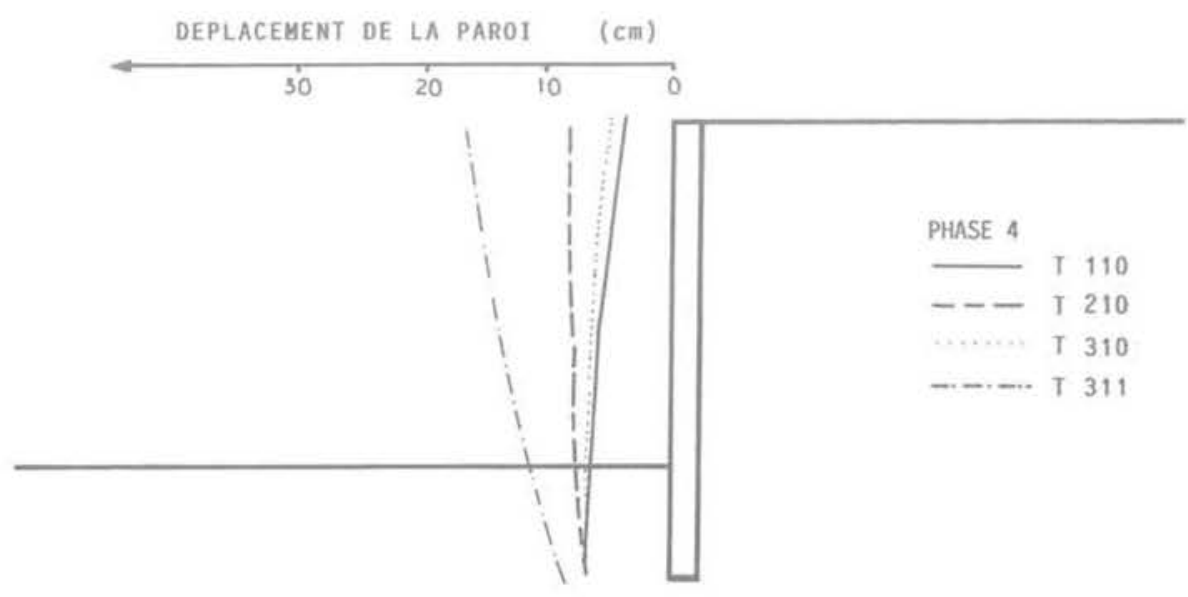

FiG.3 Déplacements de la paroi.

Wall displacements. 


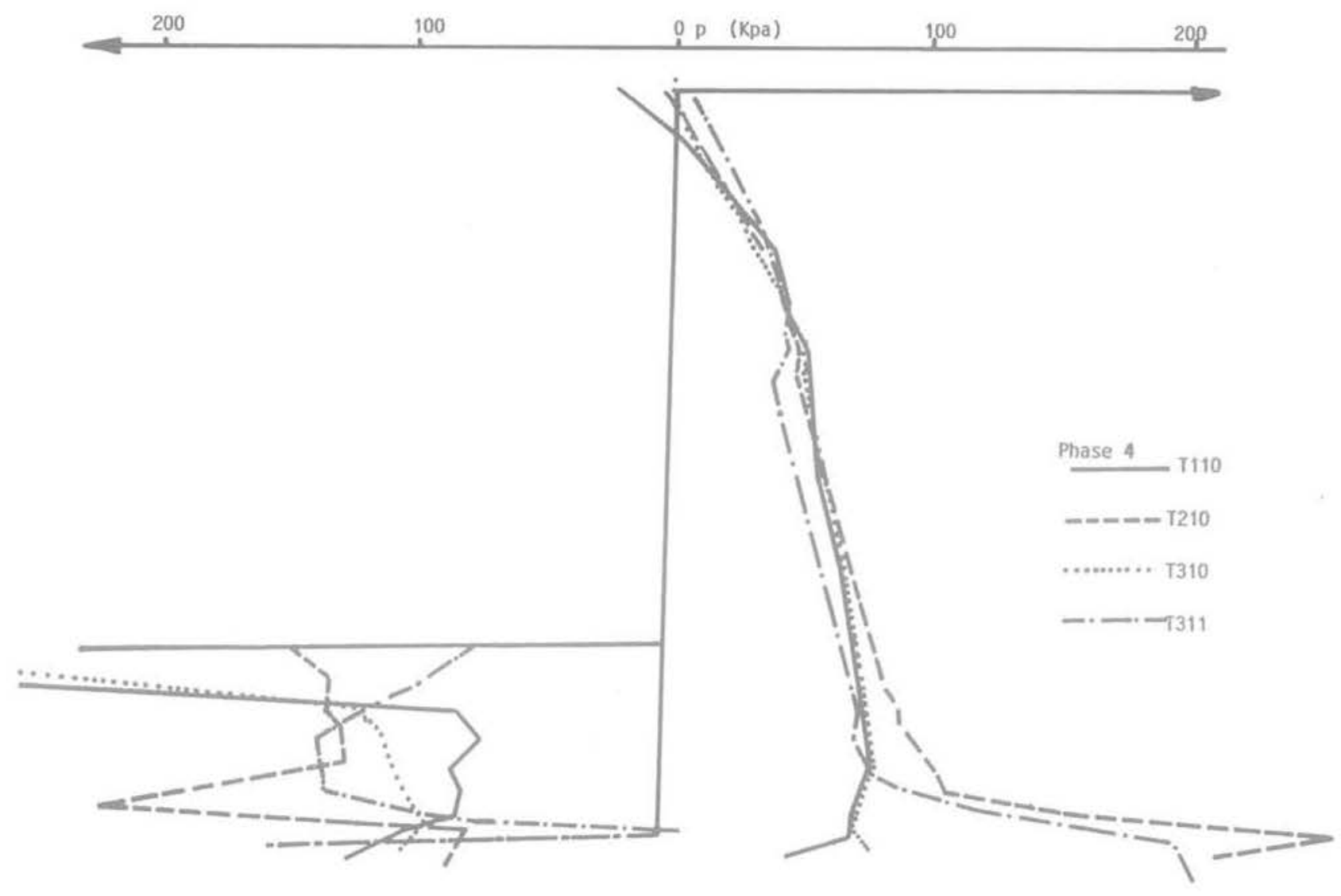

FG. 4 Pressions de contact. Contact pressures.

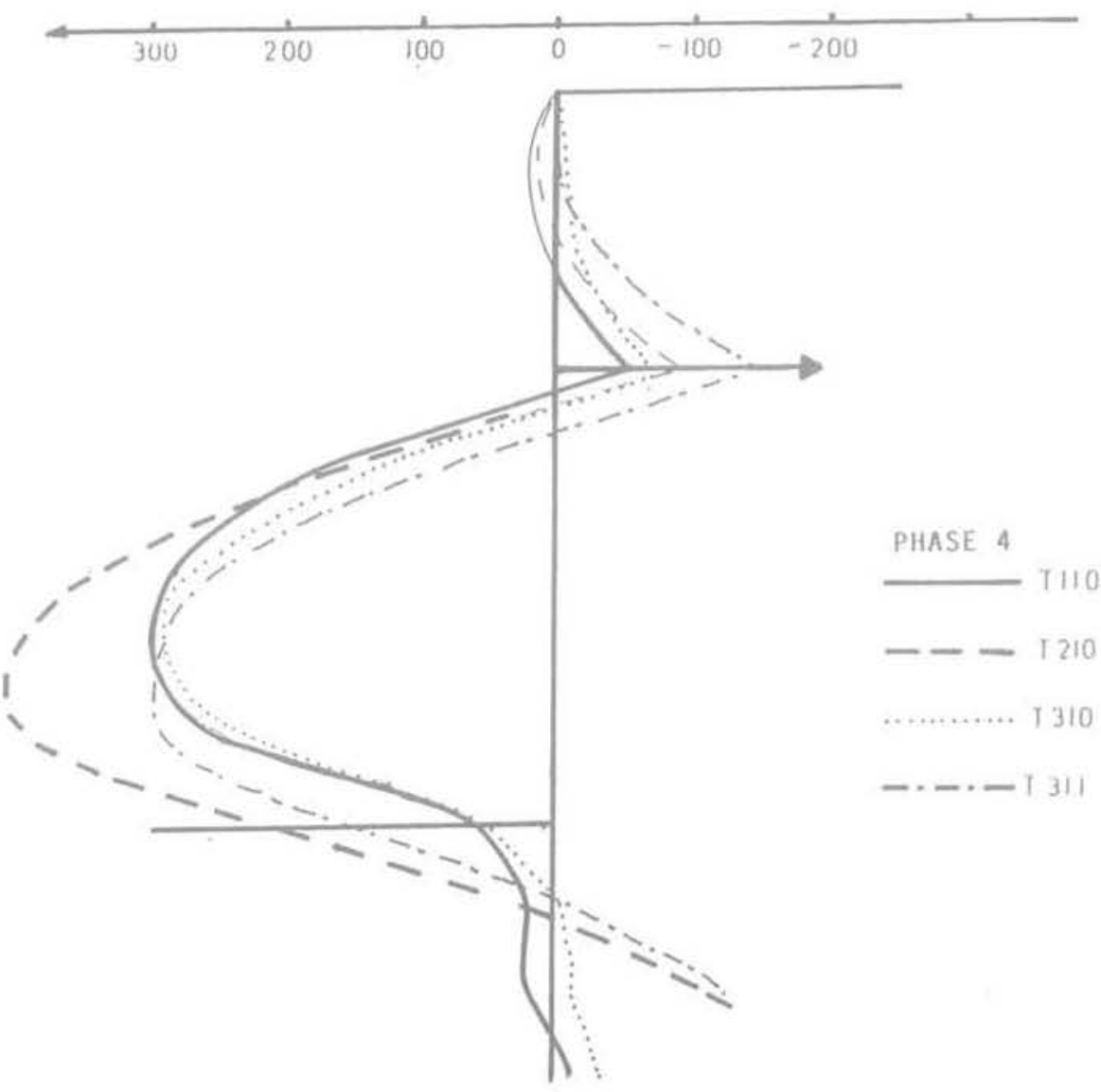

FG. 5 Moments fléchissants. 

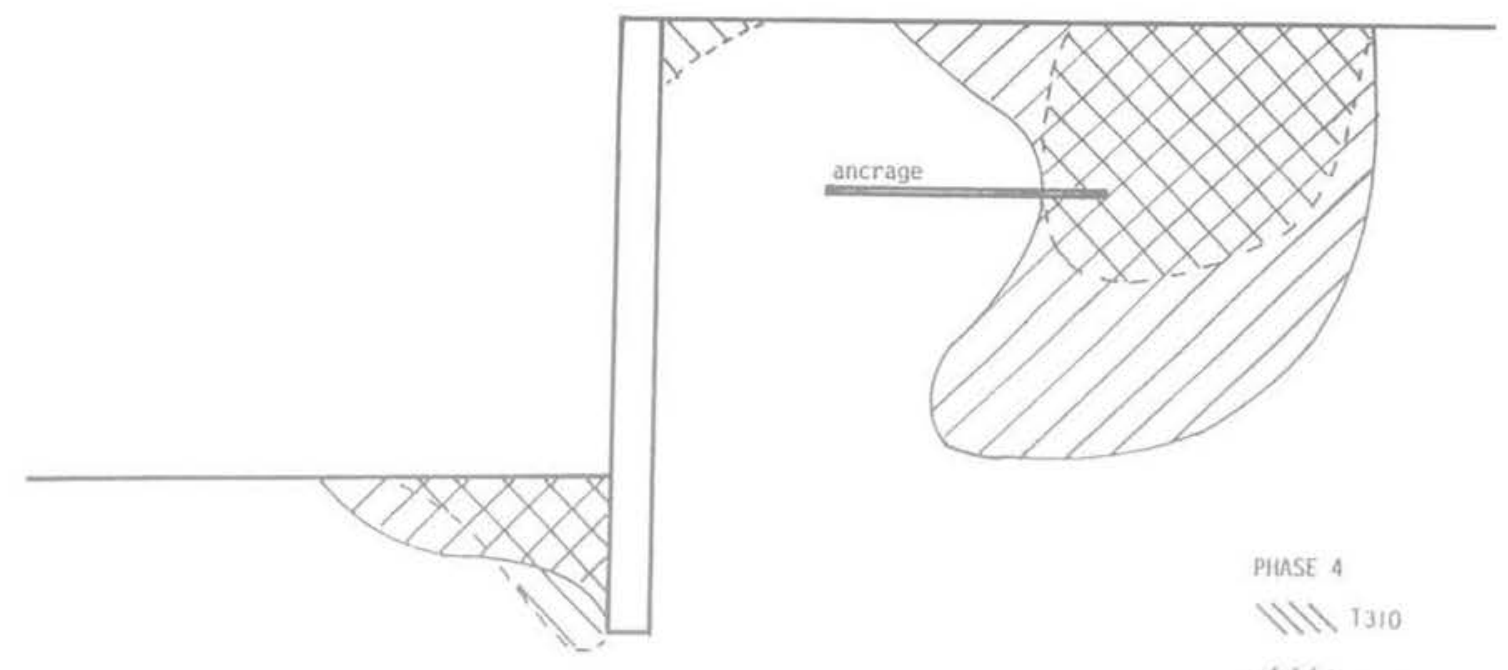

PHASE 4

MN 1310

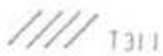

79.6 Zone de sol plastifiée.

Zones of plastified soil.

dans laquelle :

- M est le moment fléchissant maximal,

- F, la force horizontale appliquée en tête de la paroi, et

- $\lambda$, un facteur sans dimension.

Le terme $\left(\left(E_{,} I_{0} / E_{s}\right)^{1 / 3}\right.$ a la dimension d'une longueur dans un modèle bidimensionnel et il est noté $I_{0}$ :

$$
I_{0}=\left(E_{p} I_{p} / E_{s}\right)^{1 / 3}
$$

L'équation de Winkler s'écrit :

$$
\frac{\partial^{4} y}{\partial z^{4}}-\frac{K}{E_{p} I_{p}} y=0
$$

dans laquelle :

- K est le module de réaction.

- y, le déplacement horizontal et,

- z, la profondeur.

La solution se réduit, dans le cas d'une paroi infiniment profonde à :

$y: C_{1} \sin z \Lambda_{0}{ }^{*} e^{-2 \lambda}{ }_{0}^{*}+C_{2} \cos z I_{0}{ }^{*} e^{-z / \pi}{ }_{0}^{*}$

dans laquelle

$$
1_{0} *=\left(\frac{4 E_{p} I_{p}}{K}\right)^{1 / 4}
$$

Le moment fléchissant maximal, dans le cas d'une paroi soumise à un effort horizontal en tête est donné par :

$$
M_{\max }=F 1_{0} * \frac{e^{-\pi / 4}}{\sqrt{2}}
$$

A partir des équations (1) et (5), on peut définir $\mathrm{K}$ de telle sorte que $\mathrm{I}_{0}=\mathrm{I}_{0}$ * .

On en déduit :

$$
\mathrm{K}=\mu_{\mathrm{f}} \mathrm{E}_{\mathrm{s}} / \mathrm{I}_{0}
$$

ce qui signifie que le module de réaction dépend de la rigidité de la paroi dans le cas où celle-ci est relativement souple.
Pour une paroi infiniment rigide, on obtient de même

$$
\mathrm{K}=\mu_{\mathrm{r}} \mathrm{E}_{\mathrm{s}} / \mathrm{h}
$$

$\mu_{r}$ et $\mu_{\text {f }}$ sont des coefficients sans dimension.

L'équation 7 est semblable à celle présentée par Terzaghi (2)

\section{3.}

\section{Validité de l'hypothèse de Winkler}

La concordance entre les moments fléchissants calculés selon la méthode du module de réaction et ceux obtenus par le modèle d'éléments finis ne signifie pas que I'hypothèse de Winkler soit pertinente.

D'une manière générale, il n'y a pas proportionnalité entre les déplacements et les pressions obtenues par le modèle aux éléments finis, excepté dans la phase de mise en tension des tirants (phase 3).

On peut observer au contraire que lors des phases de terrassements (phases 2 et 4 ), les déplacements dépendent des conditions aux limites alors que les pressions de contact sont indépendantes de celles-ci.

Les pressions de contact sol/paroi sont seulement associées à la courbure de la paroi et il est nécessaire, soit de dissocier « le déplacement d'ensemble » du mur de la « déformation propre $n$, soit d'utiliser le concept du déplacement relatif.

\section{2}

\section{Méthode du déplacement relatif}

Les principes de cette méthode ont été présentés par Marche [1] pour des pieux soumis à des déplacements horizontaux de sol, en raison de remblaiements sur des terrains compressibles par exemple.

La relation entre pression de contact et déplacement relatif peut s'écrire :

$$
p(z)=K(y(z)-g(z))
$$



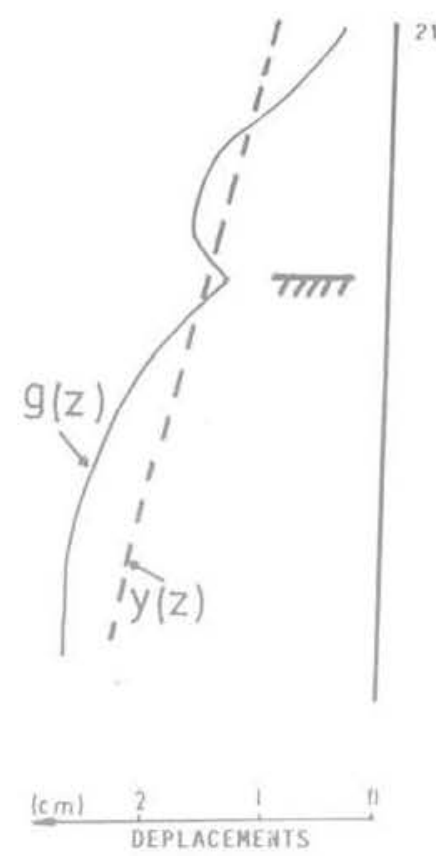

(a)

Displacements

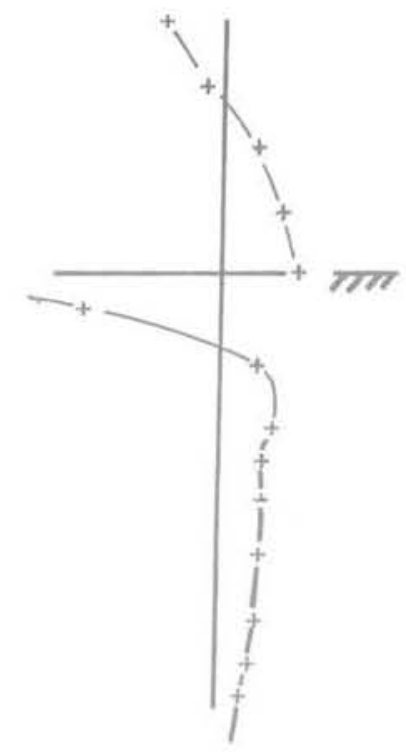

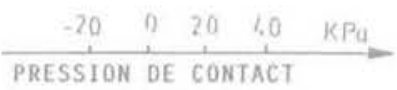

DIFFERENTIELLE (b)

Differential contact pressure

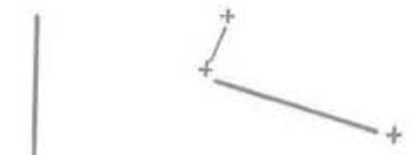

ZONE DE VARIATION RAPIDE DES DEPLACEMENTS

ET PRESSIONS

$77 \%$

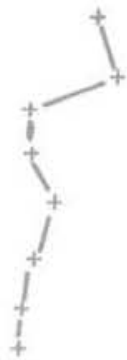

\section{$\frac{2000}{\text { 9. } 4000 \mathrm{KN} / \mathrm{M}^{3}}$}

Goeffjcjent of $\begin{aligned} & \text { DEDUIT (a) ET (b) } \\ & \text { from }\end{aligned}$

FiG. 7 Module de réaction. T Ø 001/Phase 2.

Coefficient of subgrade reaction.

dans laquelle :

- $\mathrm{p}(\mathrm{z})$ est la pression horizontale,

- y (z), la déformation de la paroi, et

- g (z), la déformation du sol en l'absence de paroi.

L'équation de Winkler devient:

$$
\frac{\partial^{4} y}{\partial z^{4}}-\frac{K(y-g)}{E_{p} I_{p}}=0
$$

A l'aide des résultats des modèles T000 et T001, on a pu obtenir une vérification de l'équation (8). Les valeurs de $K$ ainsi trouvées sont en accord avec celles issues de l'analyse des moments fléchissants (Fig. 7).

\section{3}

\section{Formulation analytique du module de réaction}

Parmi les solutions analytiques connues qui concernent les contraintes et déplacements à la surface d'un massif semi-infini ; il en est une, d'une importance particulière pour l'étude du module de réaction. Il s'agit de la distribution : $\sigma_{\mathrm{x}}=\sigma_{0} \cos \omega \mathrm{z}$ (Fig. 8).

Les équations complètes décrivant les contraintes et déplacements à l'intérieur du massif élastique sont données en annexe 1.

Le point essentiel est que cette distribution de contrainte provoque en surface du massif une déformation proportionnelle en tout point à la contrainte.

Dans ces conditions, il est légitime d'écrire:

$$
\mathrm{K}=\frac{\sigma_{\mathrm{x}}}{\mathrm{U}_{\mathrm{x}}}=\frac{\mathrm{E}_{\mathrm{s}} \omega}{\alpha}=\frac{\mathrm{E}_{\mathrm{s}} 2 \pi \mathrm{f}^{\prime}}{\alpha}
$$

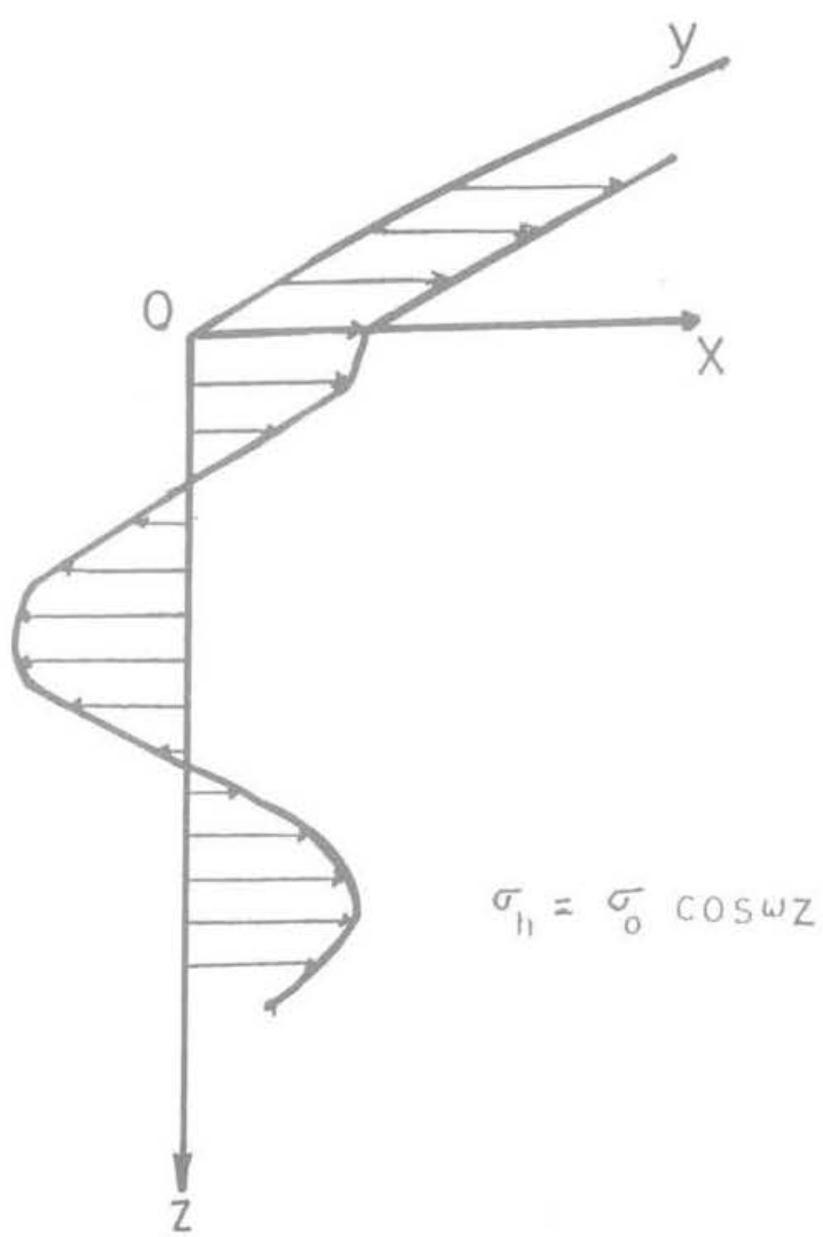

FIG. 8 Milieu élastique semi-infini, soumis à un chargement sinusoïdal.

Elastic halfspace loaded by a cosine like pressure distribution. 
- $\alpha$, est un coefficient sans dimension donné dans le tableau III,

- $\mathrm{f}$, est l'inverse d'une longueur.

TABIEAU III Valeur de $\alpha$.

\begin{tabular}{l|c|c}
\cline { 2 - 3 } Coefficient de Poisson & Lisse & Rugue de contact \\
\hline$v=0,3$ & 1,82 & 1,67 \\
\hline$v=0,5$ & 1,50 & 1,50 \\
\hline
\end{tabular}

A chaque valeur de fest associé un module de réaction K (f) qui lui est proportionnel.

Par transformation de Fourier, l'équation (9) peut s'écrire:

$$
(2 \pi f)^{4} Y+(Y-G) \frac{2 \pi f E_{5}}{\alpha E_{p} I_{p}}=0
$$

formule dans laquelle $Y$ (f) et $G$ (f) représentent les tranformées de Fourier des fonctions y (z) et g(z).

D'après la définition de $I_{0}$ (eq2) :

$$
(2 \pi f)^{3} Y+(Y-G) \frac{1}{\propto 1_{0}{ }^{3}}=0
$$

en posant :

$$
\mathrm{f}_{0}=\frac{1}{2 \pi \mathrm{l}_{0} \propto \propto^{1 / 3}}
$$

on obtient :

$$
Y=G \Delta d
$$

avec

$$
\Delta d=\frac{1}{1+\left(f / f_{0}\right)^{3}}
$$

on obtient de même les transformées de Fourier $\Pi$ (f) des contraintes p (z), ainsi que celles $\Omega$ (f) des moments fléchissants.

$$
\begin{array}{ll}
\Pi(f)=G\left[\frac{-2 \pi E_{s} f_{0}}{\propto}\right] \Delta p \text { avec } \quad \Delta p=\frac{\left(f / f_{0}\right)^{4}}{1+\left(f / f_{0}\right)^{3}} \\
\Omega(f)=G\left[\frac{E_{s}}{2 f_{0} \propto}\right] \Delta m \text { avec } \quad \Delta m=\frac{\left(f / f_{0}\right)^{2}}{1+\left(f / f_{0}\right)^{3}}
\end{array}
$$

Les opérateurs $\Delta d, \Delta p$ et $\Delta m$ ont été représentés en fonction de $\mathrm{f} / \mathrm{f}_{0}$ sur la figure 9 .

On observe que $\Delta d$ est proche de 1 pour les faibles valeurs de f. C'est-à-dire que le déplacement y est essentiellement donné par la forme générale de la courbe g. En revanche, $\Delta p$ croît rapidement avec f, c'est-à-dire que les points anguleux de g produiront des pressions de contact très grandes sur la paroi. Les moments fléchissants (opérateur $\Delta \mathrm{m}$ ) sont essentiellement influencés par les valeurs de f comprises entre $\mathrm{f}_{0} / 5$ et $5 \mathrm{f}_{0}$.

A titre d'exemple, les transformées de Fourier des pressions de contact et des moments fléchissants sont présentés sur la figure 10 pour le modèle 201, phase 4.

Les moments obtenus par double intégration des pressions de contact (c'est-à-dire en divisant $\Pi$ par $\left.-2 \pi f^{2}\right)$ sont proches de ceux fournis directement par le modèle.

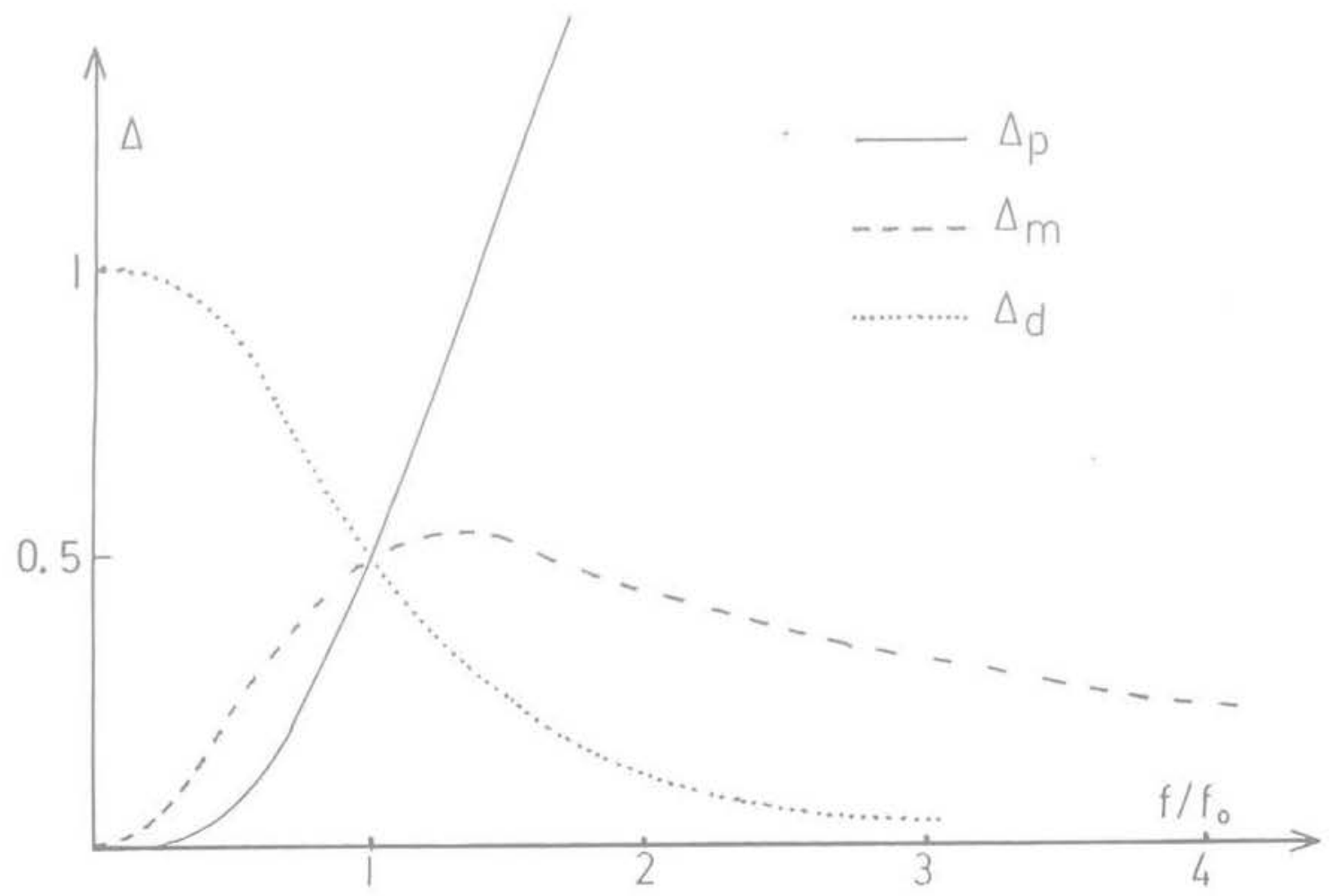

FIG.9 Facteurs de déplacements. Moments fléchissants et pressions de contact. Factors of displacements. Bending moments and contact pressure. 


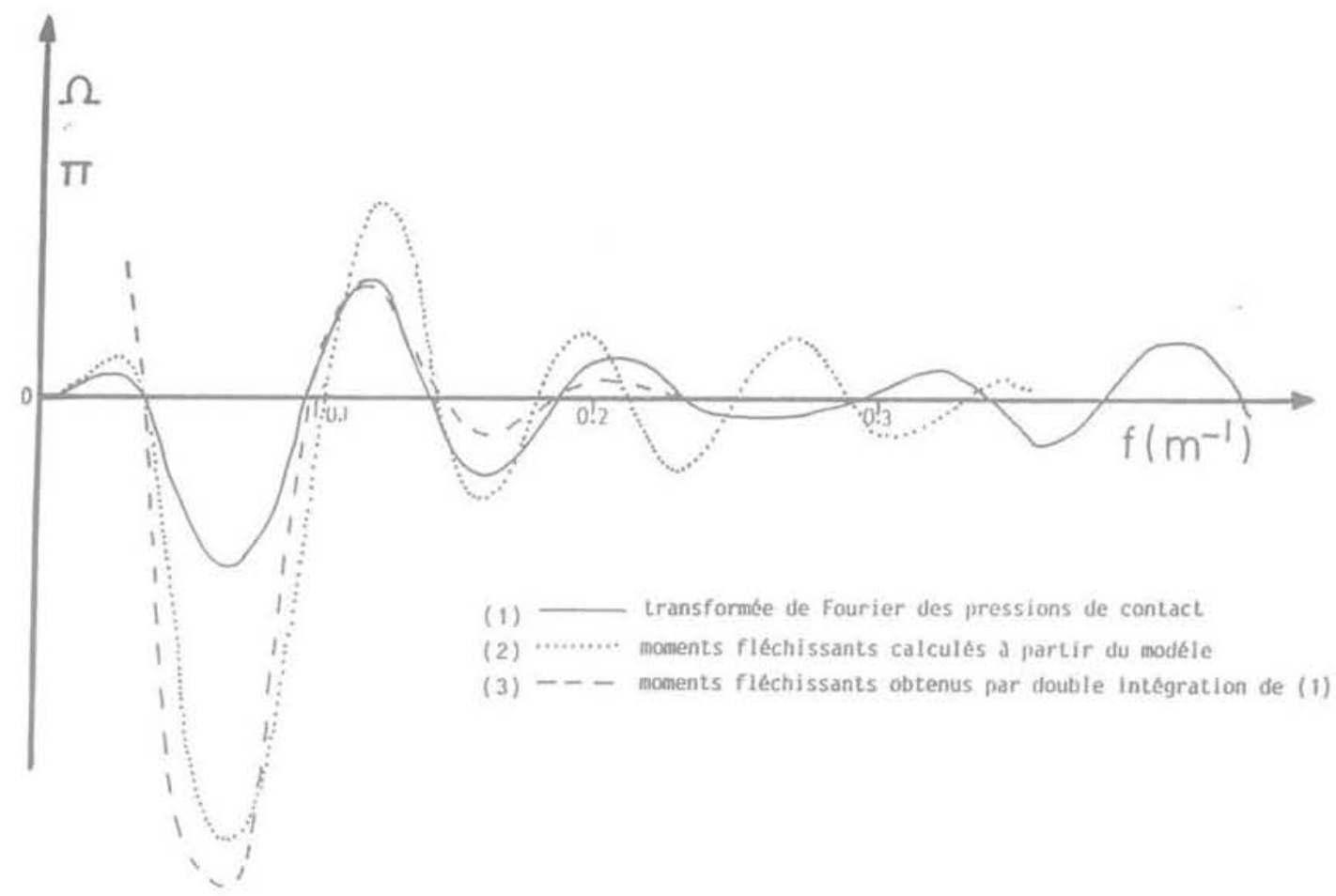

FG. 10 Transformée de Fourier des pressions de contact et des moments fléchissants (T201/Phase 4). Fourier's transform of contact pressures and bending moments.

\section{4}

\section{Plastification du sol}

La figure 6 qui présentait les zones plastifiées montrait que derrière la paroi, l'équilibre limite de poussée n'était pas atteint.

En se référant aux équations d'équilibres d'une charge de type sinusoïdal (annexe 1) on peut montrer que la contrainte déviatorique $\mathrm{D}$ :

$$
D^{2}=\frac{1}{2}\left(\sigma_{x}-\sigma_{x}\right)^{2}+\tau^{2} x y
$$

atteint un maximum pour $\mathrm{x}=\frac{1}{\omega}$ et qu'elle est nulle au contact de la paroi $(x=0)$, pour un contact sol/paroi lisse.

On peut en conclure que non seulement il n'y a pas de relation entre le maximum de pression de contact et le début de plastification du sol, mais que cette plastification ne se produit pas nécessairement au contact de la paroi.

\section{4}

\section{Conclusion}

La comparaison de deux méthodes de calcul sur un cas particulier ne peut prétendre à une complète généralité. Cependant, l'étude paramétrique d'une paroi ancrée à l'aide d'un modèle aux éléments finis a permis de dégager quelques points essentiels : les déplacements de la paroi sont liés aux limites du modèle et au type de contact sol/paroi. Les efforts dans la paroi, ainsi que les pressions de contact, sont en revanche indépendants des limites du modèle.

L'utilisation de la notion de module de réaction est cependant possible sous certaines conditions : on peut, par exemple, appliquer la notion de module de réaction au déplacement relatif sol/paroi afin de déterminer à la fois les efforts dans la paroi et les déplacements.

Enfin, l'utilisation de la transformation de Fourier permet de donner une base théorique à la notion de module de réaction. Il apparaît cependant que ce module dépend des dimensions de la paroi et de la souplesse relative de la paroi par rapport au sol.

L'apparition de zones de plasticité, côté poussée, n'est pas exactement conforme au schéma classique.

Non seulement la contrainte verticale n'est pas égale au poids des terres, mais le déviateur maximal n'apparaît pas au contact de la paroi. Il semble, de plus, qu'il n'y a pas de relation directe entre la pression de contact et le déviateur maximal.

\section{$\overline{\text { Bibliographie }}$}

Marche - Sollicitations en flexion des pieux par les couches qu'ils traversent, Ph. D. Thesis, École Polytechnique Fédérale de Lausanne, 1974

Terzaghi K. - Evaluation of coefficients of subgrade reaction, Geotechnique $5, n^{\circ} 4$, pp. 297-326, 1955. 


\section{Annexe}

\section{A.1 Contraintes et déplacements dans un milieu élastique \\ semi-infini produits par un chargement sinusoïdal}

La distribution de pression est :

$$
\sigma_{\mathrm{h}}=\operatorname{Cos} \omega \mathrm{z} \cdot \sigma_{0}
$$

\section{Contact lisse}

Pour un contact lisse : $\tau_{x z}=0$ pour $x=0$ quelle que soit la profondeur.

Les contraintes sont les suivantes:

$$
\begin{aligned}
& \sigma_{z}=\cos \omega z\left(e^{-\omega x}-w x e^{-\omega x}\right) \sigma_{0} \\
& \sigma_{x}=\cos \omega z\left(e^{-1 \omega x}+w x e^{-\omega x}\right) \sigma_{0} \\
& \tau_{x z}=\sin \omega z\left(-\omega x e^{-\omega x}\right) \sigma_{0}
\end{aligned}
$$

Les déplacements sont donnés par :

$$
\begin{aligned}
& U_{x}=\cos \omega z e^{-\omega x}\left[2 \frac{\left(1-v^{2}\right)}{\omega E_{s}}\right] \sigma_{0} \\
& U_{z}=\sin \omega z e^{-\omega x}\left[\frac{\left(1-v-2 v^{2}-(1+v) \omega x\right)}{\omega E_{s}}\right] \sigma_{0}
\end{aligned}
$$

Contact rugueux

$$
\mathrm{U}_{2}=0 \text { pour } \mathrm{x}=0
$$

En notant $\xi=1 / 2(1-v)$ on obtient pour les contraintes :

$$
\begin{aligned}
& \sigma_{z}=\cos \omega z\left[(2 \xi-1) \mathrm{e}^{-\omega x}-\xi \omega x \mathrm{e}^{-\omega x}\right] \sigma_{0} \\
& \sigma_{x}=\cos \omega z\left[\mathrm{e}^{-\omega x}+\xi \omega x \mathrm{e}^{-\omega x}\right] \sigma_{0} \\
& \tau_{x z}=\sin \omega z\left[(1-\xi) \mathrm{e}^{-\omega x}+\xi \omega x \mathrm{e}^{-\omega x}\right] \sigma_{0}
\end{aligned}
$$

Le déplacement est :

$$
\mathrm{U}_{\mathrm{x}}=\cos \omega \mathrm{z} \frac{\left(3-4 v^{2}+v\right)}{2(1-v) \omega \mathrm{E}_{\mathrm{s}}} \sigma_{0}
$$

